\title{
Multiple painless masses: periostitis deformans secondary to fluoride intoxication
}

\author{
Munish Chitkara • Paula J. Rackoff • Luis S. Beltran
}

Published online: 19 December 2013

(C) ISS 2013

\section{Diagnosis}

The differential diagnosis based on the imaging findings included ossification of subperiosteal hematomas, ectopic calcification in the setting of a connective tissue disorder, and periostitis deformans secondary to fluoride intoxication. Laboratory assays were requested by the patient's rheumatologist, which were notable for a mildly elevated alkaline phosphatase level $(216 \mathrm{U} / \mathrm{L}$, reference range $=40-115 \mathrm{U} / \mathrm{L})$ and a markedly elevated serum fluoride level $(3.9 \mathrm{mg} / \mathrm{L}$, normal range $<0.2 \mathrm{mg} / \mathrm{L}$ ), confirming the diagnosis of fluoride intoxication. The findings were discussed with the patient, who admitted to chronically "huffing" Freon, or inhaling the chlorofluorocarbon gas from a plastic bag.

\section{Discussion}

Periostitis deformans is a well-recognized but rarely seen manifestation of skeletal fluorosis associated with subacute fluoride intoxication. The classic finding, first described in 1952 [1], and further characterized in 1966 in habitual drinkers of fluoride-preserved Spanish wine, is of a nodular, exuberant, and symmetric periostitis, observed in combination with the other manifestations of skeletal fluorosis [2].

The case presentation can be found at doi: 10.1007/s00256-013-1787-0.

M. Chitkara $(\triangle) \cdot$ L. S. Beltran

Department of Radiology, NYU Langone Medical Center and

Hospital for Joint Diseases, 301 East 17th Street, New York 10003, USA

e-mail: munish.chitkara@nyumc.org

\section{P. J. Rackoff}

Rheumatology Division, Department of Medicine, NYU Langone

Medical Center and Center for Musculoskeletal Care,

New York, USA
Internationally, skeletal fluorosis is an endemic disease, observed in populations consuming fluoride-rich well water [3]. Within the United States, the disease is much less common, described in isolated case reports secondary to excess ingestion of brewed tea, toothpaste, or fluoride-containing analgesics $[4,5]$. Recently, periostitis deformans has been linked to the fluoride-containing anti-fungal medication voriconazole [6].

Once ingested, the fluoride ion substitutes for the hydroxyl ion in hydroxyapatite, resulting in fluorapatite [7]. This exchange has been shown to occur most readily at the cancellous and periosteal surfaces, i.e., areas of high turnover [8]. The fluorapatite crystal is less soluble and more stable than hydroxyapatite, resulting in greater resistance to bone remodeling. Additionally, the ion itself directly stimulates osteoblastic activity, increasing bone formation [7].

These biochemical features directly translate to what is described radiographically in skeletal fluorosis: a combination of osteosclerosis of the axial skeleton, periosteal new bone formation, ligamentous/tendinous ossification, and marginal osteophyte formation[2]. The osteosclerotic phase can progress to an osteoporotic one, which progressively involves the areas of sclerosis.

In addition to the aforementioned features of skeletal fluorosis, the pathognomonic imaging finding in periostitis deformans is the luxuriant hyperostotic periostitis resembling bone tumors, originally termed periosteal pseudotumors. These present symmetrically along the long bones of the forearm and leg and within the phalanges, typically no more than "almond-sized" in the fingers, and "apple-sized" in the forearm and leg [2].

Treatment is aimed at removing the toxic substance from the patient's environment; however, as the half-life of fluoride averages 7 years in the adult skeleton [7], resolution of osseous findings is most likely a prolonged process. A single case 
report following a patient with skeletal fluorosis for nearly a decade after stopping the offending agent showed a gradual decrease in bone density over the course of 9 years [9].

Periostitis deformans has been linked to the abuse of the inhaled anesthetic methoxyflurane [5], although not to chronic abuse of an inhaled chlorofluorocarbon. While chlorofluorocarbons and methoxyflurane are both organofluoride compounds, it is ultimately a diagnosis of exclusion that the chlorofluorocarbon is the cause of this patient's elevated serum fluoride level and osseous findings. Notably, the other known potential causes of fluoride intoxication were eliminated as sources.

In conclusion, although periostitis deformans is a rare entity, knowledge of its classic radiological appearance is important so that the radiologist can suggest the fluoric origin of the disease, prompting a search for the offending agent in the patient's environment.

Conflict of interest None.

\section{References}

1. Soriano M. Periostitis deformans. Ann Rheum Dis. 1952;11:154-61.

2. Soriano M, Manchón F. Radiological aspects of a new type of bone fluorosis, periostitis deformans. Radiology. 1966;87(6):1089-94.

3. Fewtrell L, Smith S, Kay D, Bartram J. An attempt to estimate the global burden of disease due to fluoride in drinking water. J Water Health. 2006;4(4):533-54.

4. Joshi S, Hlaing T, Whitford GM, Compston JE. Skeletal fluorosis due to excessive tea and toothpaste consumption. Osteoporos Int. 2011;22(9):2557-60.

5. Klemmer PJ, Hadler NM. Subacute fluorosis a consequence of abuse of an organofluoride anesthetic. Ann Intern Med. 1978;89:607-11.

6. Chen L, Mulligan ME. Medication-induced periostitis in lung transplant patients: periostitis deformans revisited. Skeletal Radiol. 2011;40(2):143-8.

7. Whitford GM. Intake and metabolism of fluoride. Adv Dent Res. 1994;8(1):5-14.

8. Taves DR. New approach to the treatment of bone disease with fluoride. Fed Proc. 1970;29(3):1185-7.

9. Kurland ES, Schulman RC, Zerwekh JE, Reinus WR, Dempster DW, Whyte MP. Recovery from skeletal fluorosis (an enigmatic, American case). J Bone Miner Res. 2007;22(1):163-70. 